Juliana Luporini do Nascimento ${ }^{1}$

Everardo Duarte Nunes ${ }^{1}$

${ }^{1}$ Faculdade de Ciências Médicas, Universidade Estadual de Campinas. Cidade Universitária Zeferino Vaz, Distrito de Barão Geraldo. 13.083-970 Campinas SP Brasil.

jluporini10@hotmail.com

\section{Quase uma auto/biografia: um estudo sobre os cientistas sociais na saúde a partir do Currículo Lattes}

\author{
Almost an autobiography: a study of social scientists in health \\ based on the Lattes Curriculum
}

Abstract Among the various ways of adopting the biographical approach, we used the curriculum vitaes (CVs) of Brazilian researchers who work as social scientists in health as our research material. These CVs are part of the Lattes Platform of $\mathrm{CNPq}$ - the National Council for Scientific and Technological Development, which includes Research and Institutional Directories. We analyzed $238 \mathrm{CVs}$ for this study. The CVs contain, among other things, the following information: professional qualifications, activities and projects, academic production, participation in panels for the evaluation of theses and dissertations, research centers and laboratories and a summarized autobiography. In this work there is a brief review of the importance of autobiography for the social sciences, emphasizing the CV as a form of "autobiographical practice." We highlight some results, such as it being a group consisting predominantly of women, graduates in social sciences, anthropology, sociology or political science, with postgraduate degrees. The highest concentration of social scientists is located in Brazil's southern and southeastern regions. In some institutions the main activities of social scientists are as teachers and researchers with great thematic diversity in research.

Key words Autobiography, Curriculum vitae, Social scientists, Health, Lattes curriculum
Resumo Dentro das diversas maneiras de utilizar a abordagem biográfica, adotamos como material de pesquisa o Curriculum vitae $(\mathrm{CV})$ de pesquisadores brasileiros que atuam como cientistas sociais da saúde. Estes CV fazem parte da Plataforma Lattes do CNPq (Conselho Nacional de Desenvolvimento Científico e Tecnológico), que inclui os Diretórios de Pesquisa e das Instituições. Neste trabalho são analisados $238 \mathrm{CV}$. Os CV contêm, entre outras, as seguintes informações: formação profissional, atividades e projetos, produção acadêmica, participação em bancas, núcleose laboratórios de pesquisa, pequena autobiografia. Neste trabalho há breve revisão sobre a importância da auto/biografia para as ciências sociais, enfatizando o CV como uma forma de "prática autobiográfica”. Destacamos como resultados: grupo formado predominantemente por mulheres, graduadas em ciências sociais, antropologia, sociologia ou ciência política, com pós-graduação; maior concentração de cientistas sociais nas regiões sul e sudeste; e em algumas instituições; atuação principal como docentes e pesquisadores; grande diversidade temática nas pesquisas.

Palavras-chave Auto/biografia, Curriculum vitae, Cientistas sociais, Saúde, Currículo Lattes 


\section{Introdução}

Os recentes e crescentes interesses pela biografia levaram Merril e West ${ }^{1}$ a escreverem que Esta é uma época da biografia, e contar estórias (stories) parece onipresente na cultura popular: nós consumimos as estórias de celebridades, somos fascinados por estórias na TV, e somos constantemente fascinados com as narrativas de guerra, como pode ser testemunhado pelas várias séries que se repetem na televisão.

Mas, não é somente a cultura popular que está impregnada pela onda biográfica, girando em termos de celebridades. Há como revisam as autoras, um avanço da abordagem biográfica $\mathrm{em}$ diversos e até mesmo surpreendentes contextos, por exemplo, biografias do universo e das cidades.

Sem dúvida, os métodos biográficos tornaram-se ponto de referência nos ambientes acadêmicos embora algumas vezes marginalizados e contestados. Esta é uma questão ainda presente e compartilhada por pesquisadores de diversos países, como Brasil e Argentina, com referência à reflexão sobre o gênero biográfico nas universida${d e s^{2}}^{2}$. Apesar desta ressalva, o chamado retorno da biografia, vem acontecendo de diversas maneiras: publicações (livros e artigos, capítulos de livros), associações, grupos de trabalho, revistas digitais, congressos, seminários.

Sem dúvida, esta importância pode ser vista quando pesquisadores como Chamberlayne et al. ${ }^{3}$ intitulam seu livro de The Turn to Biographical Methods In Social Sciences, mostrando que houve uma mudança na abrangência e influência deste tema nas ciências sociais, tanto na orientação das disciplinas, como nas inter-relações entre elas.

Ao relatarem a trajetória deste biographical turn, os autores associam o desenvolvimento dos métodos biográficos com a história da teoria social. Para eles, a adoção de relatos pessoais varia entre as disciplinas, sendo que os historiadores desde há muito tempo faziam uso de testemunho pessoal, ao passo que os sociólogos valorizavam os dados quantitativos, muito embora, como lembram os autores ${ }^{3}$, nas origens da sociologia os diários, as cartas, as fotografias e as histórias de vida foram reconhecidos como fonte típica de materiais pelos pesquisadores do século XIX e início do século XX. Sem dúvida, a principal referência é a de Thomas e Znaniecki que no início do século vinte relataram as experiências do camponês polonês que imigrou para os Estados Unidos, dando início a uma intensa atividade de investigação quando se formou um grupo de pes- quisadores no que se denomina a $1^{\text {a }}$. Escola de Chicago.

Segundo Chamberlayne et al. ${ }^{3}$, nos anos 1980, a utilização da história de vida como um instrumento emancipador foi feita pelo serviço social, ao passo que na sociologia da medicina os métodos interpretativos é que são utilizados. Citam, ainda, a forte presença da abordagem biográfica nas pesquisas em gerontologia. Sem dúvida, é nos anos 1990 que essa abordagem tornou-se amplamente aceita. Os autores referem-se em especial ao desenvolvimento na Grã-Bretanha, sendo que na França e Alemanha os antecedentes do movimento biográfico são do início dos anos 1970. Lembre-se que nos anos 1980, Bertaux ${ }^{4}$ publicou importantes trabalhos tratando da abordagem biográfica.

A expressão approche biographique é criação de Bertaux ${ }^{5}$ e "constitui un pari sur l'avenir uma aposta para o futuro. Nesse texto, o autor analisa as diferenças entre récits de vie que corresponde em inglês a life stories e histoires de vie, que corresponde a life histories. Lembra que Denzin ${ }^{6}$ propôs uma distinção "que me parece devemos retomar" entre life story e life history. Para Denzin life story é a história contada pela própria pessoa e life history estudos sobre uma dada pessoa e compreende não somente seu relato, mas também outros documentos como testemunhos, história clínica, etc., sendo, para o autor, superior a life story.

Na década de 1980 houve um crescente interesse sobre a questão e o aumento de volume das publicações foi se adensando no movimento em torno da abordagem biográfica, culminando com a inauguração de um campo que passou a ser denominado de sociologia biográfica. Roberts e Kyllonen ${ }^{7}$ assim definem esse novo campo: em termos gerais, pode-se dizer que é uma tentativa de compreender as experiências e perspectivas de mudança dos indivíduos em suas vidas diárias, o que eles veem como importante, e como fornecem interpretações relatos sobre seu passado, presente e futuro.

Aqui, os autores, como outros que revisam a abordagem biográfica sempre se referem a Charles Wright Mills quando ele, entre os oito preceitos e cuidados a serem tomados em um projeto de pesquisa sociológica, recomenda: Os problemas da ciência social, quando formulados adequadamente, devem incluir, tanto as preocupações como as questões, a biografia e a história, e o âmbito de suas relações complexas. Dentro deste âmbito, a vida do indivíduo e a evolução das sociedades ocorrem; e dentro desse âmbito a imaginação 
sociológica tem sua possibilidade de influir na qualidade da vida humana de nossa época8.

Esse alerta é importante, pois como dizem Roberts e Kyllonen ${ }^{7}$ a ênfase no biográfico em sociologia não é um reducionismo à vida individual, mas o importante é a interação da biografia, história e estrutura como ensina Mills8.

Foi, também, tomando esta referência a Mills que Shantz ${ }^{9}$ estranha que a discussão sobre a utilização da biografia na sociologia não tenha tido o mesmo vigor que se encontra na antropologia, literatura e história. O próprio autor acrescenta que $O$ impulso recente para uma volta à sociologia biográfica pode ser encontrada na crise pós-moderna da representação, e diz que, Destacados pensadores da Continental Philosophy e críticos da teoria social argumentam que a auto/biografia desempenha um papel fundamental na construção e no desenvolvimento tanto dos significados individual e cultural, como no engajamento político e econômico. Citando Taylor e Littleton ${ }^{10}$, escreve que teóricos pós-modernos desafiaram análises simplistas e não críticas nas quais as pessoas eram reduzidas a simples categorias de identidade demográfica (por ex. mulher, negro, classe trabalhadora), posicionando-as dentro de uma presumivel estrutura social ${ }^{9}$. Para o autor, A sociologia biográfica levanta importantes questões de identidade, pertencimento, voz, conhecimento e poder e o lugar da ciência social ortodoxa em relação a esses assuntos .

\section{Porque "Quase uma auto/biografia"}

Como vimos anteriormente, a abordagem biográfica tem sido ampliada variando o seu significado de acordo com o enfoque e tipo de pesquisa (como já situava Bertaux ${ }^{5}$ no início dos anos 1980, de l'unité à la diversité), mas conservando sempre a perspectiva de ao transcender as estruturas evidenciar a presença do sujeito.

Assim, compreendemos a importância da história de vida profissional captada (ou que se tenta captar) no calor da entrevista, ou de um relato circunstanciado autobiográfico, mas acreditamos também que há formas de aproximação que permitem traçar um perfil, não um retrato acabado dos sujeitos investigados. Dessa forma, ao lado da história oral e dos depoimentos pessoais, podemos utilizar, por exemplo, como é o caso deste trabalho, o currículum vitae (no texto usamos de forma abreviada CV).

Embora já tivesse sido utilizado por Miller e Morgan $^{11}$ e referendado por Roberts ${ }^{12}$ há poucas referências da sua aplicação para a aborda- gem biográfica, apesar de ser universal o uso acadêmico do CV. Como salientam Miller e Mor$\operatorname{gan}^{11}$, isso amplia o campo das atividades convencionais denominadas pelos termos biografia e autobiografia. Para eles, o CV pode ser entendido como uma forma de prática autobiográfica, alguém centralmente envolvido com a construção e apresentação do self em um contexto ocupacional especial.

A riqueza de aspectos levantados pelos autores sobre o CV e sua teorização a partir do momento em que este instrumento se torna essencial para as carreiras acadêmicas não podem ser marginalizados. Tomando como referência Go$\mathrm{ffman}^{13} \mathrm{e}$ suas ideias de presentation of self e lembrando que este autor "estava interessado nos encontros face-a-face" abre uma especial oportunidade de aplicar suas ideias em um conceito de "indicadores de desempenho" (performance indicators). Da mesma forma, da tradição etnometodológica são derivadas as noções de "procedimentos narrativos e indexicalidade" (accounting procedures and indexicality), assim como as derivadas da distinção entre "narrativas temáticas" (thematic narratives) - grau de maior racionalidade, claramente orientadas para certos objetivos e as "narrativas rapsódicas" (rhapsodic narratives) - aparentemente menos racionais, menos dirigidas. Sintetizando as orientações derivadas de Goffman feitas por Miller e Morgan ${ }^{11}$ anotamos como o sujeito deve expor seu CV:

1. Apresentar-se como um sincere performer e não de maneira que seja inaceitável ou inapropriada pelos padrões societários;

2, Estabelecer e manter um front (informações e dados) detalhado e não trivial;

3. Dar espaço para comunicações menos comuns e rotineiras (dramatic realization);

4. Respeitar a cultura acadêmica fornecendo informações que se situem além dos aspectos quantitativos e qualitativos, no sentido goffmaniano de idealization;

5. Incluir ou não determinados itens, como marital status, por exemplo;

6. Não deturpar os fatos, ou seja, incluir, por exemplo, fictitous articles, books;

7. Não mistificar aspectos fora do que é rotinizado no $\mathrm{CV}$;

8. Apresentar-se como um acadêmico, portador de um academic self, membro de uma comunidade.

Os autores de forma enfática escrevem Esperamos ter demonstrado que a reflexão sobre a produção e avaliação de currículos não é simplesmente uma questão de interesse paroquial, mas levanta 
a possibilidade para a exploração de toda uma série de práticas auto/biográficas ${ }^{11}$.

Embora não tenhamos a pretensão de adotar na íntegra a excelente reflexão feita pelos autores, ela serve como um ponto especial de referência para a nossa pesquisa que toma os CVs como material documental - quase uma autobiografia - na medida em que retomamos os CVs/ autobiográficos e os submetemos a uma análise biográfica - da história profissional pessoal contada pelos cientistas sociais à história coletiva contada por nós - uma "biografia coletiva". Nesse sentido, nos aproximamos de Merril e West ${ }^{1}$. Para eles O termo auto/biografia foi cunhado para chamar a atenção para o inter-relacionamento entre a construção que alguém faz da própria vida ainda que autobiográfico e a construção da vida [feita] por um outro através da biografia Explicam que há um movimento entre as duas estórias - a do sujeito e de quem a escreve,

De outro lado, a originalidade deste estudo prende-se ao fato de que embora existam vários estudos sobre os cientistas sociais ${ }^{14-16}$, há poucos estudos que circunscreveram determinados grupos de cientistas sociais, por exemplo, aqueles que trabalham na área da saúde. Nos Estados Unidos as primeiras pesquisas foram feitas por Straus ${ }^{17}$, e prosseguiram ao longo dos anos; entre nós, podem ser citados os estudos de Campos e Nunes ${ }^{18}$, Barros e Nunes ${ }^{19}$ e mais recentemente Nascimento ${ }^{20}$ que utilizou o Currículo Lattes como fonte de informação.

\section{O Currículo Lattes}

Neste trabalho, como assinalamos acima, adotamos como principal fonte de dados o Currículo Vitae da Plataforma Lattes do CNPq.

O CV Lattes é um componente da Plataforma Lattes desenvolvido pelo $\mathrm{CNPq}$ adotado pelo Ministério de Ciência e Tecnologia, FINEP, CA$\mathrm{PES} / \mathrm{MEC}$ e usado pela comunidade científica, como um sistema de informação curricular. Conforme o $\mathrm{CNPq}^{21} \mathrm{O}$ Currículo Lattes se tornou um padrão nacional no registro da vida pregressa e atual dos estudantes e pesquisadores do país, e é hoje adotado pela maioria das instituições de fomento, universidades e institutos de pesquisa do País. Por sua riqueza de informações e sua crescente confiabilidade e abrangência, se tornou elemento indispensável e compulsório à análise de mérito e competência dos pleitos de financiamentos na área de ciência e tecnologia.

No final dos anos 1980 o CNPq possuía mais de 30.000 currículos em suas bases. Mas foi so- mente no início dos anos 90 que começaram a ser cadastrados os currículos através de um formulário eletrônico. Hoje são cerca de 1.779.778 currículos, na base de dados da instituição,

Digianpietri et al. ${ }^{22}$ ao realizarem uma ampla análise de 1.236.548 currículos, baixados em maio de 2011, trazem importantes informações das quais destacamos algumas a seguir. Em relação às grandes áreas do conhecimento, os pesquisadores distribuem-se da seguinte forma: ciências humanas (19\%), ciências sociais aplicadas (18\%), ciências da saúde (17\%), ciências exatas e da terra (13\%), ciências biológicas (9\%), engenharias (95), ciências agrárias (7\%), linguística, letras e artes (7\%), outras (1\%); cada pesquisador informou 2,6 áreas de interesse.

\section{A pesquisa sobre os cientistas sociais}

Como foco de interesse deste trabalho, buscamos conhecer, a partir dos currículos Lattes, a configuração do campo dos profissionais que atuam na área da saúde coletiva e que referem alguma formação de graduação ou pós-graduação nas ciências sociais, sociologia, antropologia ou ciência política. Entendemos que esta seria uma estratégia adequada para informar a biografia de uma área, com base nas biografias individuais descritas no currículo.

A busca dos CVs junto à Plataforma Lattes foi realizada no ano de 2010 durante três meses. O curto espaço de tempo da busca deve-se ao fato de ser uma Plataforma "viva", constantemente alimentada pelos sujeitos que a integram. Foi visitado um total de 3181 currículos e, com base nos critérios de inclusão da pesquisa, selecionados 238 currículos.

Foram selecionados sujeitos com doutorado e com formação de graduação e/ou pós-graduação nas ciências sociais, antropologia, sociologia, ou ciência política, com atuação profissional (pesquisa, extensão ou docência) na área da saúde.

Dos 238 currículos selecionados, 128 tem formação de graduação nas áreas das ciências sociais e pós-graduação em ciências sociais e/ou em outros campos de conhecimento; 110 profissionais tem graduação em diferentes áreas do conhecimento e formação de pós-graduação (mestrado e/ou doutorado) nas áreas das ciências sociais. O interesse pelo estudo das biografias dos profissionais que optaram em aliar as ciências sociais à saúde, a partir dos currículos da Plataforma, remete à necessidade estrutural do conhecimento do campo híbrido da saúde coletiva, mas também, do conhecimento da relação 
estrutural do campo com as trajetórias individuais, com as autobiografias profissionais.

\section{O que relatam os CVs Lattes}

Optamos por dividir o material em três etapas: a) uma descrição correspondente às variáveis sexo, ano e área de graduação e pós-graduação; b) distribuição geográfica: localização das cidades e instituições de formação de graduação e pós-graduação e os locais e tipos de vínculos profissionais; c) áreas de atuação no campo científico: áreas de trabalho e principais linhas de pesquisa. Com base nesta divisão do material configuramos a identidade de sujeitos que, a partir de escolhas individuais, se juntam em coletividades e se distribuem em espaços geográficos distintos.

Dentre os currículos selecionados há uma maioria do sexo feminino $(65,5 \%)$ em comparação aos do sexo masculino (34,5\%). Tal dado relaciona-se a um elemento de extrema importância e que confere uma característica específica ao campo, o fato da escolha da área profissional ter uma grande influência pelo recorte de gênero. Existe uma tendência de que as áreas das humanidades e da saúde concentrem um maior número de mulheres, fato evidenciado por Velho e Léon $^{23}$ e que se relaciona com a socialização e a incorporação de papéis sociais distintos que acabam por incentivar meninos e meninas a diferentes escolhas profissionais.

Verificamos isso claramente ao analisarmos, a partir dos nossos dados, as relações entre os cursos de graduação e o sexo. O número de mulheres em cursos como o de ciências sociais (69) é mais do que o dobro do número de homens (31), e em cursos como a medicina é quase igual, sendo 13 mulheres para 11 homens, o que referenda o processo de feminização da área médica e, principalmente, o fato de que em áreas da saúde a proporção é igual ou levemente superior dos homens em relação às mulheres ${ }^{24}$.

Além de o campo ter como marca o recorte de gênero ele configura-se como multidisciplinar composto mesmo que em menor número, por profissionais com formação de graduação ou pós-graduação, em áreas muito distintas como direito, engenharia civil, física. As áreas de maior concentração, na graduação são: as ciências sociais (128), a medicina (24), a psicologia (17), a enfermagem (9). No mestrado algumas áreas se mantêm, como a psicologia (11) e as ciências sociais (26) e suas respectivas áreas disciplinares como a antropologia (50), a ciência política (11) e a sociologia (45), que passam a ter uma maior expressão em relação à graduação, e áreas como a educação (10) e a saúde coletiva (35) que agora compõem as etapas de formação dos profissionais. No doutorado os cursos com maior expressão são respectivamente: saúde coletiva (63); antropologia (56) e sociologia (52). A relação entre os cursos de graduação e pós-graduação denotam o percurso de formação realizado pelos profissionais que optaram pelas ciências sociais em sua formação e se profissionalizaram em direção ao campo da saúde, mais especificamente da saúde coletiva. É expressiva a concentração nestas áreas no decorrer da sua profissionalização em comparação à multidisciplinaridade encontrada em maior número na graduação.

Escolhas relacionadas aos diferentes cursos na perspectiva de uma profissionalização segue um percurso que busca as instituições de maior prestigio. Apesar dos incentivos governamentais buscando ampliar o quadro das Universidades Federais em diferentes localidades brasileiras, este estudo traz como caracterização principal uma distribuição geográfica assimétrica.

A distribuição desigual dos programas de pós-graduação, como referendado no Plano Nacional de Pós-Graduação (2005-2010) ${ }^{25}$, pôde ser visualizada nos nossos dados. Percebemos uma trajetória dos profissionais, no decorrer da sua formação, em direção, aos Estados de São Paulo; Rio de Janeiro; Rio Grande do Sul; Minas Gerais; Bahia, de forma geral, cartograficamente, na direção leste do mapa. Regiões como Maranhão, Pará; Amazonas que tem de uma a três titulações de graduação, não apresentam titulações de pós-graduação. Outros estados como Roraima, Mato Grosso, Mato Grosso do Sul, Acre, Tocantins, não apresentam nenhuma titulação em nível de graduação e pós-graduação. Esse quadro em que há uma concentração dos programas de pós-graduação na região sudeste do Brasil foi constado por Barata ${ }^{26}$, em que cita um aumento dos programas de pós-graduação em saúde coletiva e uma concentração de $66,3 \%$ na região sudeste.

A institucionalização do profissional é um importante marco na configuração do campo, no que podemos chamar de uma espécie de biografia coletiva que buscamos construir ao evidenciar a trajetória de formação. Configura-se como a escolha pela área, pelo lugar e pelo tipo de trabalho a ser desenvolvido. Nesse sentido, dentre os diversos tipos de vínculo profissional, há um grande número de servidores públicos, $70,6 \%$, em sua quase totalidade em Universidades Públicas Federais e Estaduais. Estes locais re- 
presentam, para muitos a consolidação da carreira acadêmica por serem ambientes legitimados socialmente como produtores do conhecimento, aliando a pratica de pesquisa à docência.

A forma como o profissional ingressa na instituição corresponde à forma como ele se institucionaliza, esta pode ser através da docência e/ ou pesquisa. Assim, a profissionalização e a institucionalização são processos concomitantes ao se profissionalizar inicia-se um longo trajeto na aprendizagem do habitus social e individual ${ }^{27}$ específicos da área e da instituição escolhida. A construção do habitus social e individual consiste no modo como a sociedade é compreendida e como as pessoas compreendem a sua autoimagem, sendo fruto da relação entre o "eu e o nós". Tal perspectiva permite estender as redes de relações entre os sujeitos e as organizações sociais, no caso em estudo, as instituições universitárias.

Há um número expressivo de professores (171) e de pesquisadores (46), em relação ao total dos profissionais. Lembramos que além das atividades docentes e de pesquisa, uma característica do trabalho institucional é o exercício de uma diversidade de atividades incluindo as administrativas, consultoria, conselhos, coordenações e etc.

Em nosso trabalho $17 \%$ dos profissionais exercem atividades na extensão, a participação em conselhos chega a $48 \%$ e em áreas de coordenadoria e/ou administrativas $46 \%$, a participação em núcleos e laboratórios de pesquisa corresponde a $33 \%$, atividade de pesquisa chega a $87 \%$ e o ensino na graduação e pós-graduação corresponde respectivamente a $54 \%$ e $56,5 \%$.

A pesquisa configura-se como a principal função expressa pelos profissionais no currículo. Quando analisamos as linhas temáticas visualizamos uma multiplicidade de temas, condizentes com a estrutura hibrida que constitui o campo da saúde coletiva ${ }^{28-30}$. Há muitas linhas citadas apenas por um pesquisador, como: saúde e religião; pensamento social brasileiro; consumo e cultura; relação médico/paciente, entre outros. As linhas temáticas mais citadas foram: gênero, saúde reprodutiva e sexualidade (16\%); HIV/ AIDS (3,4\%); saúde mental (4,2\%); política pública $(5,0 \%)$; violência $(5,5 \%)$; população indígena $(6,3 \%)$ e trabalho $(7,6 \%)$.

A identificação dos temas de interesse dos profissionais fornece traços importantes para construirmos as suas biografias e entender quanto a biografia individual se relaciona com a biografia coletiva, como elementos que se constroem mutuamente. As escolhas temáticas relacio- nam-se com os locais de vinculo profissional, com os temas de interesse de determinados grupos, com os incentivos financeiros e com o contexto sociocultural no qual estão inseridos. Por exemplo, a temática indígena é trabalhada prioritariamente em Estados como o Amazonas, Roraima e Mato Grosso. De outro lado, na década de 2000, a violência emergiu como uma importante temática por configurar um dos principais problemas sociais e se tornou referência para muitos pesquisadores. Da mesma forma, os estudos de gênero constituem uma importante temática para o campo da saúde tornando-se cada vez mais proeminente e destacando-se em termos institucionais e organizacionais.

Embora a localização temática do pesquisador seja um importante referencial em sua biografia, devemos considerar que no plano mais geral das suas escolhas a Plataforma Lattes possibilita que ele se situe em uma grande área, em uma área de atuação e em uma subárea.

Do total de dados coletados nesta pesquisa as ciências da saúde compreendem $44,2 \%$ como grande área, e as ciências humanas 50\%; relacionado à área de atuação a saúde coletiva totaliza $37,4 \%$, já os que se referem à antropologia, à sociologia e à ciência política correspondem 42,4\% dos profissionais. Um fato interessante é que os graduados na área de ciências sociais, genericamente, não incluem essa área como de atuação, mas sim a antropologia e a sociologia.

Um dado encontrado que nos elucida quanto a importância da relação entre a profissionalização e a identidade profissional, consiste no fato de que 14 profissionais não referem a área da saúde ou a saúde coletiva como grande área e área de atuação, respectivamente, apesar de estarem inseridos em instituições das áreas da saúde mantém sua identidade ancorada nas ciências sociais.

\section{Considerações finais}

Consideramos que a utilização do CV mostrouse adequada para o estudo realizado, sendo que as informações do Currículo Lattes prestam-se a diversas modalidades de estudos. No caso desta pesquisa, os dados permitiram traçar um perfil, delineando os principais traços de um grupo de cientistas sociais que atuam no campo da saúde. Um retrato mais acabado poderá ser obtido com os relatos de vida, especialmente, no que se refere à profissionalização quando escolhem a saúde como interface em sua formação e atividade, usando outras técnicas como entrevistas, 
relatos orais, depoimentos. Como é citado por Roberts ${ }^{12}$, o CV é a construção de uma estória que relata uma situação particular em vez de alguma ideia mais ampla de 'verdade.'

Dentro da proposta inicial, o objetivo deste trabalho foi alcançado, ou seja, partindo de uma autobiografia - a curricular - revelar algumas características de um grupo, no sentido que constituem um coletivo com características que o identificam no cenário geral dos cientistas sociais. Mais próximos ou mais distantes de suas identidades primeiras, já formalizaram suas presenças em salas de aulas e nas pesquisas; hoje, estão procu- rando novos cenários de atuação, fora da academia, como pode ser visto em pesquisa recente ${ }^{31}$.

Em um próximo trabalho será analisada de forma detalhada a produção científica desses cientistas sociais nos últimos cinco anos. Pretende-se, assim, situar no campo interno da ciência sociológica como os cientistas apropriam e utilizam as matrizes sociológicas analisando os quatro principais polos de uma pesquisa: o teórico, o epistemológico, o técnico e o morfológico ${ }^{32}$. Sem dúvida, essa complementação aponta para um retrato mais fiel dos cientistas sociais dando maior visibilidade a sua identidade profissional.

\section{Colaboradores}

JL Nascimento e ED Nunes participaram igualmente de todas as etapas de elaboração do artigo. 


\section{Referências}

1. Merril B, West L. Using biographical methods in social research. Los Angeles: Sage; 2009.

2. Aguiar J. Gênero é ainda pouco valorizado na academia brasileira. Folha de São Paulo 2011; 17 dez.

3. Chamberlayne P, Bornat J, Wengraf T. The turn to biographical methods in social science: comparative issues and examples. New York: Routledge Inc.; 2000.

4. Bertaux D, editor. Biography and society: the life history approach in the social sciences. Londres: SAGE; 1981.

5. Bertaux D. L'Approche biographique: sa validité méthodologique, ses potentialités. Cah. int. sociol. 1980; 69:197-221.

6. Denzin NK. The Research Act. Chicago: Aldine; 1970.

7. Roberts B, Kyllonen R. Editorial Introduction: Special Issue - Biographical Sociology. QSR- Qualitative Sociology Review 2006; 2(1).

8. Mills CW. A imaginação sociológica. Tradução de Waltensir Dutra. Rio de Janeiro: Zahar Editores; 1965.

9. Shantz J. Biographical sociology: struggles over an emergent sociological practice. $a / b$ : Auto/Biography Studies 1990; 14(1):113-128.

10. Taylor S, Littleton K. Biographies in talk: a narra-tive-discursive research approach. $Q S R$ Qualitative Sociology Review 2006; 2(1):22-28.

11. Miller NE e Morgan D. Called to account: the CV as an autobiographical practice. Sociology 1993; 27(1):133-143.

12. Roberts B. Sociological lives and auto/biographical writing. In: Horrocks C, Milnes K, Roberts B, Robinson D, organizadores. Narrative, memory and life transitions. Huddersfield: University of Huddersfield; 2002. p. 163-170.

13. Goffman E. A representação do eu na vida cotidiana. Petrópolis: Vozes; 1983.

14. Bonelli MG. Identidade profissional e mercado de trabalho dos cientistas sociais: as ciências sociais no sistema das profissões [tese]. Campinas (SP): Unicamp; 1993.

15. Schwartzman S. As ciências sociais brasileiras no século 20. Ciência Hoje 2000; [Internet]. [acessado 2012 out 3]. Disponível em: http://www.schwartzman. org.br/simon/10mais.htm

16. Meceli S, organizador. História das ciências sociais no Brasil. 2a ed. São Paulo: Editora Sumaré; 2001.

17. Straus R. The development of a social science teaching and research program in a medical center. In: Straus R. Meeting American Sociological Society. Pensilvania: American Sociological Society; 1955.

18. Campo RZ, Nunes ED. O ensino das Ciências Sociais nas escolas profissionais na área da saúde no Brasil. Rev Saude Publica 1976; 10(4):383-390.

19. Barros NF, Nunes ED. Sociologia, medicina e a construção da sociologia da saúde. Rev Saude Publica 2009; 43(1):169-175.
20. Nascimento JL. Uma (con) figuração social: cientistas sociais, antropólogos, sociólogos e cientistas politicos em saúde no Brasil [tese]. Campinas: Unicamp; 2011.

21. Conselho Nacional de Desenvolvimento Científico e Tecnológico (CNPq). Sobre a Plataforma Lattes. [Internet]. [acessado $2014 \mathrm{fev} 25]$. Disponível em: http://www.cnpq.br/web/portal-lattes/sobre-aplataforma, 2012.

22. Digiampietri LA, Mena-Chalco JP, Pérez-Alcázar JJ; Tuesta EF; Delgado KV, Mugnaini R, Silva GS. Minerando e caracterizando dados de Currículos Lattes. Proceedings of BraSNAM, 2012. [Internet]. [acessado 2013 jun 5]. Disponível em: http:// professor.ufabc.edu.br/ jesus.mena/publications/ pdf/digiampietri2012bdLattes.pdf

23. Velho L, Léon E. A construção social da produção científica por mulheres. Cadernos PAGU 1998; (10):309-344.

24. Encontro discute a participação das mulheres nas ciências. Agência Brasil 2010; 2 fev. [Internet]. [acessado 2010 jun 20]. Disponível em: http://www. inovacaotecnologica.com.br/noticias/noticia.php? $\operatorname{artigo}=$ encontro-discute-participacao-mulheresciencias\#.UwyA4aGPLIU

25. Plano Nacional de Pós-Graduação (PNPG) - 20052010. Resumo Executivo. RBPG 2005; 2(3):185-198.

26. Barata RB. A pós-graduação e o campo da saúde coletiva. Physis 2008; 18(2):189-214.

27. Elias N. A sociedade dos indivíduos. Rio de Janeiro: Jorge Zahar; 1994.

28. Minayo MCS. Pós-graduação em Saúde Coletiva no contexto do desenvolvimento científico e tecnológico do Brasil. Cien Saude Colet 2010; 15(4):18941895.

29. Canesqui AM. Temas e abordagens das ciências sociais e humanas em saúde na produção acadêmica de 1997 a 2007. Cien Saude Colet 2010; 15(4):19551966.

30. Nunes ED, Ferreto LE, Barros NF. A pós-graduação em Saúde Coletiva no Brasil: trajetória. Cien Saude Colet 2010; 15(4):1923-1934.

31. Barros NF. Os cientistas sociais no Sistema Único de Saúde. Campinas: 2013; 158 p. (mimeo).

32. Bruyne P, Herman J, Schoutheete M. Dinâmica da Pesquisa em Ciências Sociais - os pólos da prática metodológica. Rio de Janeiro: Livraria Francisco Alves; 1991.

Artigo apresentado em 24/07/2013

Aprovado em 29/08/2013

Versão final apresentada em 09/09/2013 